\title{
Polyneuropathy in Impaired Glucose Tolerance: Is Postprandial Hyperglycemia the Main Culprit? A Mini-Review
}

\author{
N. Papanas D. Ziegler \\ Institute for Clinical Diabetology, German Diabetes Center at Heinrich Heine University, Leibniz Center for Diabetes \\ Research, and Department of Metabolic Diseases, University Hospital, Düsseldorf, Germany
}

\section{Key Words}

Diabetes mellitus $\cdot$ Hyperglycemia $\cdot$ Impaired glucose tolerance $\cdot$ Peripheral neuropathy

\begin{abstract}
There is accumulating evidence for the mutual relationship between peripheral neuropathy and impaired glucose tolerance (IGT). The key factor in the pathogenesis of neuropathy in IGT is postprandial hyperglycemia, which induces increased oxidative stress, endothelial dysfunction, and activation of both protein kinase $C$ and the polyol pathway, leading to impaired neuronal metabolism and DNA damage. Other pathogenic factors include dyslipidemia and the metabolic syndrome. The cornerstone of management is improved glycemic control, although a long sustainable effect has not been documented yet, calling for further supportive trials. Secondary therapeutic targets encompass hypolipidemic and antihypertensive treatment, smoking cessation and weight loss. The increasing awareness of peripheral neuropathy in IGT is expected to improve healthcare provision in subjects with this condition. Copyright $\odot 2012$ S. Karger AG, Basel
\end{abstract}

\begin{tabular}{ll}
\hline KARGER & ( ) 2012 S. Karger AG, Basel \\
0304-324X/13/0593-0193\$38.00/0 \\
$\begin{array}{l}\text { Fax +4161306 1234 } \\
\begin{array}{l}\text { E-Mail karger@karger.com } \\
\text { www.karger.com }\end{array}\end{array}$ & $\begin{array}{l}\text { Accessible online at: } \\
\text { www.karger.com/ger }\end{array}$
\end{tabular}

\section{Introduction}

Distal sensorimotor polyneuropathy is regarded as the most frequent diabetic peripheral neuropathy and a common chronic complication of diabetes [1]. It is linked to increased mortality and represents a key factor in the pathogenesis of diabetic foot ulceration [1]. Interestingly, it has been recognized that peripheral neuropathy may sometimes develop already in the phase of impaired glucose tolerance (IGT) [2]. The latter may easily be diagnosed by an oral glucose tolerance test (OGTT) [plasma glucose $140-199 \mathrm{mg} / \mathrm{dl}(7.8-11.0 \mathrm{mmol} / \mathrm{l}) 2 \mathrm{~h}$ following the administration of $75 \mathrm{~g}$ glucose] [3]. Alternatively, glycated hemoglobin $\left(\mathrm{HbA}_{\mathrm{lc}}\right)$ 5.7-6.4\% might be used to diagnose IGT [3]. This option appears simpler and more attractive; however, it may detect impaired glucose metabolism but cannot distinguish IGT from impaired fasting glucose (IFG) [fasting plasma glucose $100-125 \mathrm{mg} / \mathrm{dl}$ (5.6-6.9 mmol/l)] [3]. Moreover, the laboratory method of the Diabetes Control and Complications Trial must be used to assess $\mathrm{HbA}_{1 \mathrm{c}}$, and this is, currently, not always readily available, limiting its widespread application. Indeed, there is accumulating evidence that the frequency of IGT is elevated in patients with allegedly idiopathic peripheral neuropathy who are 
referred to specialized centers [4]. In a different but complementary setting, there are data suggesting that the prevalence of neuropathy and neuropathic pain in subjects with IGT lies between that of patients with normal glucose metabolism and that of patients with overt diabetes mellitus [5-7]. More recently, post-challenge hyperglycemia has been identified as a risk factor for peripheral neuropathy in the general population [8]. The aim of this review is to provide an outlook of current evidence on the relationship between peripheral neuropathy and IGT.

\section{IGT in Subjects with Peripheral Neuropathy: The Evidence from Tertiary Care Centers}

More than 10 years ago, it was realized that IGT was not infrequent among subjects with idiopathic peripheral neuropathy (with or without pain) who were referred to specialized neuropathy clinics [2,4]. Studies on such subjects have found that their glucose metabolism was commonly impaired. When an OGTT was carried out, approximately, 1 out of 3 or 1 out of 4 patients had IGT [2, 4]. This test emerged as the most sensitive to reveal impaired glucose metabolism in such patients $[2,4]$. The authors compared the frequency of IGT in their series to that reported in the general population and concluded that it was much higher in the former than the latter $[2$, 4]. A control group was included in only one of these studies, which reported a $24.5 \%$ frequency of IGT in patients with idiopathic chronic axonal polyneuropathy, as compared to $10.2 \%$ in controls, but this difference was not statistically significant after adjustment for age and $\operatorname{sex}[9]$.

These studies advanced our understanding of early neuropathic changes during the natural history of glucose metabolism. Nonetheless, three important caveats were recognized $[2,4]$. First, the frequency of IGT was either reported as generally high or compared with historical controls from much older population studies with much wider age ranges. Secondly, patients were highly selected by referral to tertiary care centers. Moreover, patient series were small and, worse still, OGTT was not always performed in all patients, further reducing the number of evaluable subjects. Thus, it was felt that the relationship of peripheral neuropathy with IGT should be enquired in population-based studies as well [2].

\section{Peripheral Neuropathy in Subjects with IGT: The Evidence from Population Studies}

Ziegler et al. [5-7] have examined peripheral neuropathy and neuropathic pain in the population-based KORA (Cooperative Research in the Region of Augsburg) study. Importantly, an OGTT was performed in all controls [5]. Overall, the frequency of neuropathy was $13 \%$ in IGT, falling between diabetes (28\%) and controls (7.4\%) [5]. Neuropathic pain was as frequent as $8.7 \%$ in IGT versus $1.2 \%$ in controls [6]. Turning to subjects with prior acute myocardial infarction, the frequency of neuropathic pain was $14.8 \%$ in IGT versus $21 \%$ in diabetes and $3.7 \%$ in controls [7]. The Hoorn study [10] also provided evidence for neurologic deficits (absent Achilles tendon reflexes, reduced vibration perception using a tuning fork at the hallux) in IGT, findings lying between diabetic and normal subjects.

More recently, Bongaerts et al. [8] have focused on the relationship between IGT and peripheral neuropathy. They examined 1,100 subjects aged 61-82 years in the population-based KORA F4 Survey (2006-2008) for polyneuropathy defined as bilateral impairment of vibration perception (tuning fork), and/or of pressure sensation (10-gram monofilament) in the feet [8].

In their work, the prevalence of polyneuropathy was $23.9 \%$ in subjects with combined IFG and IGT (IFGIGT), impressively as high as in known diabetes (22.0\%) while it was $14.8 \%$ in isolated IGT [8]. Among pre-diabetes subgroups, IFG-IGT, but not isolated IFG and IGT, was associated with a higher risk of polyneuropathy, compared to normal glucose tolerance. A J-shaped relationship was shown between polyneuropathy and quartiles of 2-hour post-challenge glucose (fig. 1), but not with fasting glucose and $\mathrm{HbA}_{1 \mathrm{c}}$ levels [8].

By contrast, some studies have not found an increased prevalence of neuropathy in IGT [2]. The recent prospective Olmsted County Impaired Glucose Survey [11] assessed neuropathy in subjects with newly diagnosed diabetes, as well as in those with pre-diabetes (IGT or IFG) and controls. They studied both typical distal symmetrical sensorimotor polyneuropathy and atypical peripheral neuropathy, the latter including small-fiber sensory or autonomic nerve fiber involvement. Using a stricter definition, the prevalence of neuropathy was $7.8 \%$ in diabetes, $1.7 \%$ in pre-diabetes and $2 \%$ in controls. Using a broader definition, the corresponding percentages were 17.4, 12.6 and $12.7 \%$, respectively [11]. The reasons for these apparently discordant findings are unclear, but they could be due to different diagnostic procedures (e.g. clinical ex- 

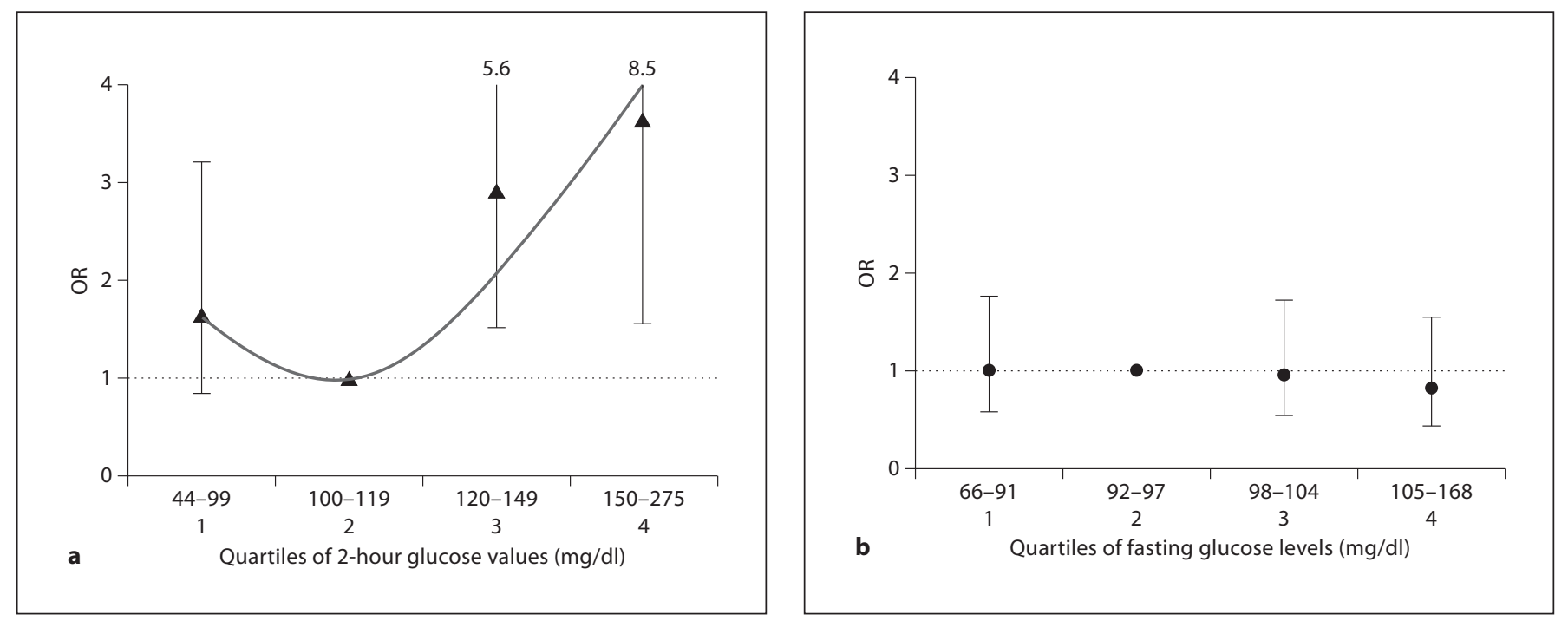

Fig. 1. J-shaped relationship between quartiles of 2-hour postload glucose (a) but not fasting glucose (b) and the presence of clinical distal sensorimotor polyneuropathy in the general population undergoing screening for pre-diabetes or diabetes by an OGTT: adjusted for age, sex, height, waist circumference, diastolic blood pressure, physical activity, and alcohol intake (based on data from reference [8]).

amination confirmed by nerve conduction studies vs. clinical criteria only), divergent demographic features and sample size of the populations studied. Perhaps it is also worth noting that neither this work nor the KORA surveys included skin biopsy in the diagnostic workup of neuropathy.

\section{Pathogenesis: A Case for Postprandial Hyperglycemia}

The cardinal etiological pathway to the development of neuropathy in subjects with IGT appears to be hyperglycemia [2]. In the more recent publication from the KORA population study, the third and fourth quartiles of 2-hour post-challenge glucose were independent risk factors of peripheral neuropathy [odds ratio (OR): 2.91, 95\% confidence interval (CI): 1.51-5.62; OR: 3.64 , 95\% CI: 1.56-8.48, respectively] [8]. In real life, subjects with IGT may develop intermittent hyperglycemia, mainly in the postprandial phase, but this is constantly repeated [3]. There is ample experimental but also clinical evidence now that acute transient hyperglycemia may generate oxidative stress and endothelial dysfunction [2, 12], ultimately resulting in impaired mitochondrial function, perturbed cellular metabolism and direct deoxyribonucleic acid (DNA) damage at the neuronal level [2]. A re- cent experimental study in dogs [13] has demonstrated that acute post-meal hyperglycemia may lead to reduced arterial flow-mediated dilatation along with increased serum methylglyoxal concentration. The latter is a highly reactive dicarbonyl intermediate metabolite of glucose that is recognized to induce oxidative stress $[13,14]$. In another recent experimental work in mice, serum methylglyoxal has been demonstrated to be associated with diminished nerve conduction velocity, depolarization of sensory neurons and mechanical and thermal hyperalgesia [14]. When hyperglycemia becomes more chronic, its deleterious effect is enhanced by activation of protein kinase $\mathrm{C}$ and of the polyol pathway, which leads to further neuronal damage [2].

A second very important pathogenic mechanism is dyslipidemia $[2,4]$. There is ample experimental evidence now to suggest that dyslipidemia exerts a substantial neurotoxic effect, which is additive to that of glucose excursions $[2,4,15]$. Mice on high-fat diet exhibit increased oxidized low-density lipoprotein (LDL) particles, which, in turn, bind to the lectin-like oxidized LDL receptor in dorsal root ganglion neurons. As a result, they activate nicotinamide adenine dinucleotide phosphate oxidase, directly inducing oxidative stress and injury [15]. In streptozotocin-induced diabetes in mice on a high-fat diet, there may be increased conversion of arachidonic acid to hydroxyeicosatetraenoic acid via the enzyme 
12/15 lipoxygenase, resulting in reduced sensory and motor nerve conduction, delayed response to pressure and thermal stimuli, as well as intra-epidermal nerve fiber loss $[2,4]$. In clinical practice, it is relevant that Hughes et al. [9] identified elevated serum triglycerides as the major risk factor for chronic idiopathic axonal neuropathy.

Moreover, other features of the metabolic syndrome and microvascular dysfunction have been implicated as well $[2,4]$. Indeed, subjects with idiopathic neuropathy have been reported to exhibit a significantly higher number of further attributes of the metabolic syndrome other than high glucose (predominantly obesity and dyslipidemia) than those without neuropathy, implicating the metabolic syndrome per se in the development of neuropathy $[2,4]$. Abdominal obesity also emerged as a risk factor for neuropathy among subjects from the general population in the KORA survey [5-7]. The contributory role of obesity appears to be exerted via its association with dyslipidemia and via increased production of inflammatory cytokines. The latter exert a dual neurotoxic effect: directly and indirectly through increased oxidative stress $[2,4]$. Finally, capillary abnormalities in sural nerve biopsies and diminished acetylcholine-induced endothelium-dependent vasodilatation in the skin have been identified before the development of neuropathy in IGT and have been implicated in its pathogenesis $[2,4]$.

\section{Peripheral Neuropathy in IGT: Clinical Manifestations}

Most patients with IGT have a less severe form of peripheral neuropathy than those with overt diabetes [2, 4]. Indeed, specialized examinations have shown fewer abnormalities in nerve conduction attributes and in intra-epidermal innervation among the former than among the latter $[2,4]$. Should there be symptoms, they include burning and/or tingling pain in the distal parts of the lower extremities $[2,4]$. On most occasions, however, patients are asymptomatic or have only very minor complaints [2]. Physical examination may reveal sensory deficits (increased vibration perception and hot/cold perception thresholds, reduced sensory nerve conduction attributes) with relative sparing of motor modalities (i.e. muscular strength and motor nerve conduction attributes) [2]. Characteristically, neuropathy in IGT involves small rather than large fibers because the former are more susceptible to the toxic effect of hyperglycemia [2].

\section{Peripheral Neuropathy in IGT: How to Diagnose}

The diagnosis of neuropathy in IGT is based on careful clinical examination $[2,16]$. As in diabetes, this should include both motor and sensory modalities, both small- and large-fiber function tests. Bedside tests include evaluation of tendon reflexes, vibration perception at the hallux by a $128-\mathrm{Hz}$ graded tuning fork, hot/cold perception, light touch and pain perception and 10-gram monofilaments to assess pressure perception $[2,16]$. Further examinations include vibration perception threshold at the hallux (measured in volts with a neurothesiometer) and quantitative temperature perception. If needed, confirmation may be sought by nerve conduction study or by skin biopsy to quantify intra-epidermal nerve fiber loss [2]. Symptoms (numbness, causalgia, sharp or dull pain, mainly in the distal parts of the lower extremities) should not be ignored [2]. Finally, corneal confocal microscopy, which quantifies corneal nerve fibers in the human eye in vivo, and Neuropad, a simple adhesive indicator test evaluating sweat gland secretory function on the plantar aspects of the feet via a color change from blue to pink, both represent useful modalities to assess small-fiber function and are emerging as potentially useful diagnostic adjuncts in neuropathy associated with IGT $[17,18]$.

\section{Treatment: Correction of Hyperglycemia and Other Factors}

Given that postprandial hyperglycemia is the main pathogenic factor, optimized glycemic control is the mainstay of treatment $[2,4]$. However, evidence for its long-term beneficial effect is still lacking. In a pioneer work, Smith et al. [19] included 32 subjects with IGT who had predominantly sensory neuropathy and some degree of neuropathic pain. They were mostly obese, had elevated serum lipids and led a sedentary lifestyle [19]. Subjects were offered detailed individualized advice to improve diet and start exercise: the aim was to achieve at least a $7 \%$ weight loss and gradually increase exercise at home to 150 min per week. Neuropathy was evaluated by a comprehensive examination including clinical assessment (Michigan Neuropathy Screening Instrument), visual analog pain scale, quantitative sensory testing, nerve conduction study, quantitative sudomotor axon reflex testing to measure sweat secretion, and skin biopsy. At 12 months, the subjects attained significant improvements in body weight, serum glucose

Papanas/Ziegler 
and cholesterol [19]. These were accompanied by significant improvements in some neurological parameters. Specifically, intra-epidermal nerve fiber density in the thighs and sweat secretion in the feet rose significantly. The change in intra-epidermal nerve fiber density correlated with improvements in peroneal nerve motor conduction velocity $(\mathrm{p}<0.004)$ and sural sensory conduction velocity $(\mathrm{p}<0.03)[19]$. At the same time, a nonsignificant increase in intra-epidermal nerve fiber density in the feet and a nonsignificant reduction in pain intensity were observed [19]. This improvement notwithstanding, sustainable effects could not be documented at 36 months. Indeed, by that time, only the reduction in serum lipids persisted, while the improvements previously achieved in neurological parameters had reversed meanwhile [20].

Equally discouraging are the results of the China Da Qing Diabetes Prevention Outcome Study [21]. This trial randomized 577 adults with IGT to a control, a diet intervention, an exercise intervention and a diet plus exercise intervention group. This treatment was offered for 6 years and the effect on the development of microvascular complications was assessed after 20 years [21]. Regrettably, neuropathy was examined by the 10 -gram monofilament only, which is insensitive to early neuropathic changes. At the end of the 20 -year period, 542 (94\%) subjects were evaluable. In the patients receiving intervention treatment, the frequency of neuropathy was not reduced in comparison to the control group (8.6 vs. 9.1\%, $\mathrm{p}=0.89$ ) [21]. Certainly, we should not be oblivious of the caveats of this work, notably the reliance on monofilament only, the low frequency of neuropathy events, and the potential selection bias, which is inherent in looking only at survivors. More importantly, we lack data on the prevalence of neuropathy at baseline [21], which would be vital to conclude whether neuropathy developed more frequently and/or progressed more quickly in the control group.

Against this background, the question arises why correction of hyperglycemia has hitherto failed to slow the development and/or progression of neuropathy in IGT. Apart from the difficulties in maintaining the adherence to lifestyle intervention [20], the major drawback is that no large-scale trials with a 20 -year follow-up and detailed evaluation of neuropathy (including repeat standardized clinical examination and confirmation by a nerve conduction study) have yet been conducted. Accordingly, trials with more patients and a longer followup are required to demonstrate this effect. The beneficial effect is even more difficult to show, given that the ear-

Polyneuropathy in IGT: Is Postprandial

Hyperglycemia the Main Culprit? lier patients are enrolled across the continuum of glucose dysmetabolism and potential neuropathic changes, the more sophisticated and accurate the diagnostic workup for neuropathy must be in order to detect small changes. Such elaborate diagnostic methodology is not always available, as shown in the Da Qing study [21] although this caveat does not apply to the lifestyle intervention program in the studies by Smith et al. [19] and Singleton et al. [20]. Thus, it appears that we need to increase our knowledge in this area. For instance, some antidiabetic regimens may be more efficacious than others in preserving nerve function due to potential additional neuroprotective properties, but this remains speculative and needs to be addressed in future trials. Of further note, antidiabetic treatment should perhaps not only seek long-term normoglycemia but also avoid glucose fluctuations, which is difficult to attain and control in the long run. Lastly, correction of hyperglycemia may need to be combined with therapeutic adjuncts mitigating oxidative stress [22] to achieve maximal benefit. In the authors' opinion, the paucity of evidence at the moment does not prove the absence of the beneficial effect of longterm normoglycemia, but suggests that the latter must be more thoroughly examined.

The other therapeutic targets are correction of hyperlipidemia, antihypertensive treatment and general measures, namely weight loss and smoking cessation [2, 4]. These are based on experience obtained with multifactorial intervention in diabetic neuropathy $[1,2]$. However, their efficacy has hitherto not been examined in neuropathy associated with IGT [2].

\section{Conclusions}

Peripheral neuropathy is demonstrable in a substantial proportion of subjects with IGT. The key factor for its development is postprandial hyperglycemia, which is neurotoxic via a number of mutually additive mechanisms: increased oxidative stress, endothelial dysfunction, activation of protein kinase $\mathrm{C}$ and of the polyol pathway, culminating in impairment of mitochondrial function and neuronal metabolism with direct osmotic injury and DNA damage. Other pathogenic factors include dyslipidemia and the metabolic syndrome. The cornerstone of management is improved glycemic control although a long sustainable effect has not yet been documented, calling for further supportive trials. Secondary therapeutic targets include general protective measures, notably hypolipidemic and antihypertensive treatment, smoking 
cessation and weight loss. The increasing awareness of peripheral neuropathy in subjects with IGT may be anticipated to help intensifying glycemic control and early screening for diabetic complications, thereby contributing to improved health care.

\section{Acknowledgement}

We thank Dr. Brenda Bongaerts for drafting figure 1 .

\section{Disclosure Statement}

N.P. and D.Z. have served as advisory board members for TrigoCare International, distributor of Neuropad.

\section{References}

1 Boulton AJ, Vinik AI, Arezzo JC, Bril V, Feldman EL, Freeman R, Malik RA, Maser RE, Sosenko JM, Ziegler D, American Diabetes Association: Diabetic neuropathies: a statement by the American Diabetes Association. Diabetes Care 2005;28:956-962.

2 Papanas N, Vinik AI, Ziegler D: Neuropathy in prediabetes: does the clock start ticking early? Nat Rev Endocrinol 2011;7:682-690.

-3 Tabák AG, Herder C, Rathmann W, Brunner EJ, Kivimäki M: Prediabetes: a high-risk state for diabetes development. Lancet 2012; 379(9833):2279-2290.

-4 Gordon Smith A: Impaired glucose tolerance and metabolic syndrome in idiopathic neuropathy. J Peripher Nerv Syst 2012;17(suppl 2):15-21.

5 Ziegler D, Rathmann W, Dickhaus T, Meisinger C, Mielck A; KORA Study Group: Prevalence of polyneuropathy in pre-diabetes and diabetes is associated with abdominal obesity and macroangiopathy: the MONICA/KORA Augsburg Surveys S2 and S3. Diabetes Care 2008;31:464-469.

- 6 Ziegler D, Rathmann W, Dickhaus T, Meisinger C, Mielck A; KORA Study Group: Neuropathic pain in diabetes, prediabetes and normal glucose tolerance: the MONICA/KORA Augsburg Surveys S2 and S3. Pain Med 2009;10:393-400.

-7 Ziegler D, Rathmann W, Meisinger C, Dickhaus T, Mielck A; KORA Study Group: Prevalence and risk factors of neuropathic pain in survivors of myocardial infarction with prediabetes and diabetes. The KORA Myocardial Infarction Registry. Eur J Pain 2009;13: 582-587.

8 Bongaerts BWC, Rathmann W, Kowall B, Herder C, Stöckl D, Meisinger C, Ziegler D: Post-challenge hyperglycemia is positively associated with diabetic polyneuropathy: the KORA F4 study. Diabetes Care 2012;35: 1891-1893.
$>9$ Hughes RA, Umapathi T, Gray IA, Gregson NA, Noori M, Pannala AS, Proteggente A, Swan AV: A controlled investigation of the cause of chronic idiopathic axonal polyneuropathy. Brain 2004;127:1723-1730.

10 de Neeling JN, Beks PJ, Bertelsmann FW, Heine RJ, Bouter LM: Peripheral somatic nerve function in relation to glucose tolerance in an elderly Caucasian population: the Hoorn study. Diabet Med 1996;13:960-966.

11 Dyck PJ, Clark VM, Overland CJ, Davies JL, Pach JM, Dyck PJ, Klein CJ, Rizza RA, Melton LJ 3rd, Carter RE, Klein R, Litchy WJ: Impaired glycemia and diabetic polyneuropathy: The OC IG Survey. Diabetes Care 2012; 35:584-591.

12 Heine RJ, Balkau B, Ceriello A, Del Prato S, Horton ES, Taskinen MR: What does postprandial hyperglycaemia mean? Diabet Med 2004;21:208-213.

13 Adolphe JL, Drew MD, Huang Q, Silver TI, Weber LP: Postprandial impairment of flowmediated dilation and elevated methylglyoxal after simple but not complex carbohydrate consumption in dogs. Nutr Res 2012; 32:278-284.

14 Bierhaus A, Fleming T, Stoyanov S, Leffler A, Babes A, Neacsu C, Sauer SK, Eberhardt M, Schnölzer M, Lasischka F, Neuhuber WL, Kichko TI, Konrade I, Elvert R, Mier W, Pirags V, Lukic IK, Morcos M, Dehmer T, Rabbani N, Thornalley PJ, Edelstein D, Nau C, Forbes J, Humpert PM, Schwaninger $M$, Ziegler D, Stern DM, Cooper ME, Haberkorn U, Brownlee M, Reeh PW, Nawroth PP: Methylglyoxal modification of $\mathrm{Na}(\mathrm{v}) 1.8 \mathrm{fa}-$ cilitates nociceptive neuron firing and causes hyperalgesia in diabetic neuropathy. Nat Med 2012;18:926-933.

15 Vincent AM, Hayes JM, McLean LL, Vivekanandan-Giri A, Pennathur S, Feldman EL: Dyslipidemia-induced neuropathy in mice: the role of oxLDL/LOX-1. Diabetes 2009;58: 2376-2385.
16 Putz Z, Tabák AG, Tóth N, Istenes I, Németh N, Gandhi RA, Hermányi Z, Keresztes K, Jermendy G, Tesfaye S, Kempler P: Noninvasive evaluation of neural impairment in subjects with impaired glucose tolerance. Diabetes Care 2009;32:181-183

17 Tavakoli M, Marshall A, Pitceathly R, Fadavi $\mathrm{H}$, Gow D, Roberts ME, Efron N, Boulton AJ, Malik RA: Corneal confocal microscopy: a novel means to detect nerve fibre damage in idiopathic small fibre neuropathy. Exp Neurol 2010;223:245-250.

18 Ziegler D, Papanas N, Rathmann W, Heier M, Scheer M, Meisinger C; KORA Study Group: Evaluation of the Neuropad sudomotor function test as a screening tool for polyneuropathy in the elderly population with diabetes and pre-diabetes. The KORA F4 survey. Diabetes Metab Res Rev 2012;28:592697.

19 Smith AG, Russell J, Feldman EL, Goldstein J, Peltier A, Smith S, Hamwi J, Pollari D, Bixby B, Howard J, Singleton JR: Lifestyle intervention for pre-diabetic neuropathy. Diabetes Care 2006;29:1294-1299.

20 Singleton JR, Bixby B, Feldman EL: Diet and exercise counseling alone does not prevent long term neuropathy progression in IGTN (abstract). Neurology 2007;68:A410.

21 Gong Q, Gregg EW, Wang J, An Y, Zhang P, Yang W, Li H, Li H, Jiang Y, Shuai Y, Zhang B, Zhang J, Gerzoff RB, Roglic G, Hu Y, Li G, Bennett PH: Long-term effects of a randomised trial of a 6-year lifestyle intervention in impaired glucose tolerance on diabetes-related microvascular complications: the China Da Qing Diabetes Prevention Outcome Study. Diabetologia 2011;54:300-307.

22 Ziegler D, Low PA, Litchy WJ, Boulton AJ, Vinik AI, Freeman R, Samigullin R, Tritschler H, Munzel U, Maus J, Schütte K, Dyck PJ: Efficacy and safety of antioxidant treatment with $\alpha$-lipoic acid over 4 years in diabetic polyneuropathy: the NATHAN 1 trial. Diabetes Care 2011;34:2054-2060. 